Pacific Journal of Mathematic 


\section{INVERSION OF CONDITIONAL EXPECTATIONS}

\section{J. YEH}

By its definition a conditional expectation is the RadonNikodym derivative of a finite signed measure. In this paper an inversion formula is given for recapturing $E(Y \mid X)$ as an inverse Fourier transform of the function $E\left(e^{i(u, X)} Y\right), u \in R^{n}$, where $X$ is an random vector and $Y$ is a random variable satisfying some regularity conditions.

1. Introduction. Let $(\Omega, \mathfrak{B}, P)$ be a probability space and let $X$ be a $k$-dimensional random vector on $(\Omega, \mathfrak{B}, P)$, i.e., a measurable transformation of $(\Omega, \mathfrak{B})$ into $\left(R^{k}, \mathfrak{B}^{k}\right)$ where $\mathfrak{B}^{k}$ is the $\sigma$-algebra of Borel sets in the $k$-dimensional Euclidean space $R^{k}$. Assume that the probability distribution $X$ is absolutely continuous with respect to the Lebesgue measure $m_{L}$ on $\left(R^{k}, \mathfrak{B}^{k}\right)$. For a real valued random variable $Y$ on $(\Omega, \mathfrak{B}, P)$ with $E(|Y|)<\infty$ let $E(Y \mid X)$ be the conditional expectation of $Y$ given $X$ which is given as a function on the value space $R^{k}$ of $X$. For $u \in R^{k}$ let $(u, X)=\sum_{j=1}^{k} u_{j} X_{j}$. In this paper we show that if $E\left[e^{i(u, X)} Y\right]$ is a $m_{L}$-integrable function of $u$ on $R^{k}$ then a version of $E(Y \mid X)$ is given by

$$
E(Y \mid X)(\xi)=\left(\frac{d P_{X}}{d m_{L}}(\xi)\right)^{-1} \frac{1}{(2 \pi)^{k}} \int_{R^{k}} e^{-i(u, \xi)} E\left[e^{i(u, X)} Y\right] m_{L}(d u)
$$

for $\xi \in R^{k}$ assuming that $\left(d P_{X} / d m_{L}\right)(\xi)>0$ for a.e. $\xi$ in $\left(R^{k}, \mathfrak{B}^{k}, m_{L}\right)$. (Our conditional expectation $E(Y \mid X)$ given as a function on $R^{k}$ rather than one on $\Omega$ is the "conditional expectation in the wide sense" in the terminology of [2].)

In preparation for (1.1) which is given in Theorem 2 in $\S 3$ we show in Theorem 1 in $\$ 3$ that if the characteristic function (i.e., the Fourier transform) $\varphi$ of a finite measure $\Phi$ on $\left(R^{k}, \mathfrak{B}^{k}\right)$ is $m_{L^{-}}$ integrable on $R^{k}$ then $\Phi$ is absolutely continuous with respect to $m_{L}$ on $\left(R^{k}, \mathfrak{B}^{k}\right)$ and a version of the Radon-Nikodym derivative of $\Phi$ with respect to $m_{L}$ is given by the inverse Fourier transform of $\varphi$. We base this result on the Lévy-Haviland Theorem for the inversion of Fourier transforms of finite measures on $\left(R^{k}, \mathfrak{B}^{k}\right)$.

The substance of Propositions 1,2 , and 3 in $\$ 2$ concerning conditional probabilities, conditional expectations and regular conditional distributions given as functions on the value space of $X$ is wellknown. We included them here in order to state them in a convenient form.

This research is an attempt at justifying a calculus of Wiener integral originated by M. D. Donsker. Its applications to conditional 
function space integrals will appear in a subsequent paper.

2. Integration of conditional expectations. Throughout $\S 2$ we write $(\Omega, \mathfrak{B}, P)$ for a probability space and $X$ and $Y$ for two measurable transformations of $(\Omega, \mathfrak{B})$ into two arbitrary measurable spaces $(S, \mathfrak{F})$ and $(T$, (S) respectively unless further specified. We write $P_{X}$ and $P_{Y}$ for the probability measures on $(S, \mathfrak{F})$ and $(T, \mathbb{S})$ determined by $X$ and $Y$ respectively, i.e.,

$$
P_{X}(F)=P\left(X^{-1}(F)\right) \quad \text { for } F \in \mathfrak{F}
$$

and similarly for $P_{Y}$.

Definition 1. For $G \in \mathbb{B}$ fixed, the conditional probability of $Y$ being in $G$ given $X$, written $P(Y \in G \mid X)$, is defined to be any real valued $\mathfrak{F}$-measurable and $P_{X}$-integrable function $\psi$ on $S$ such that

$$
P\left(Y^{-1}(G) \cap X^{-1}(F)\right)=\int_{F} \psi(\xi) P_{X}(d \xi) \quad \text { for } \quad F \in \mathfrak{F} .
$$

From the Radon-Nikodym Theorem follows that such a function $\psi$ always exists and is determined uniquely up to a null set of ( $S$, $\left.\widetilde{F}, P_{X}\right)$. We shall use $P(Y \in G \mid X)$ to mean either the class of all such functions $\psi$ or a particular member in it depending on the context. Thus

$$
P\left(Y^{-1}(G) \cap X^{-1}(F)\right)=\int_{F} P(Y \in G \mid X)(\xi) P_{X}(d \xi) \text { for } \quad F \in \preccurlyeq
$$

DEFINITION 2. Let $Z$ be a real valued random variable on $(\Omega$, B, $P$ ) with $E(|Z|)<\infty$. The conditional expectation of $Z$ given $X$, written $E(Z \mid X)$, is defined to be any real valued $\widetilde{F}$-measurable and $P_{X}$-integrable function $\psi$ on $S$ such that

$$
\int_{X^{-1}(F)} Z(\omega) P(d \omega)=\int_{F} \psi(\xi) P_{X}(d \xi) \quad \text { for } \quad F \in \mathfrak{F} .
$$

The same remark as the one following Definition 1 holds here too and we have

$$
\int_{X^{-1}(F)} Z(\omega) P(d \omega)=\int_{F} E(Z \mid X)(\xi) P_{X}(d \xi) \quad \text { for } \quad F \in \mathfrak{\mho} .
$$

DEFINITION 3. By the regular conditional distribution of $Y$ given $X$, written $P(Y \mid X)$, we mean a real valued function $\psi$ on (S) $\times S$ such that

$1^{\circ}$ for every $G \in \mathbb{S}, \psi(G, \cdot)$ is a version of $P(Y \in G \mid X)$

$2^{\circ}$ for every $\xi \in S, \psi(\cdot, \xi)$ is a probability measure on $(T, \mathbb{B})$. 
Thus when we need to indicate the arguments of $P(Y \mid X)$, we write $P(Y \mid X)(G, \xi)$ for $(G, \xi) \in \mathbb{B} \times S$. It is known that a function $\psi$ satisfying the conditions $1^{\circ}$ and $2^{\circ}$ of Definition 3 always exists whenever the value space $(T$, (B) of $Y$ is a Borel space and in particular when $\left(T,(S)=\left(R^{k}, \mathfrak{B}^{k}\right)\right.$. For a proof of this statement see [1]. The proof of Proposition 1 below which relates the regular conditional distribution to the conditional expectation is parallel to the proof of the corresponding theorem in which these two are given as functions on $\Omega$ rather than as functions on the value space $S$ of $X$. (See for instance Proposition 4.28 in [1].) We give the proof here for the sake of completeness.

Proposition 1. Let $f$ be a measurable transformation of (T, (B) into $\left(R^{1}, \mathfrak{B}^{1}\right)$ and $f \in L_{1}\left(T, \mathfrak{B}, P_{Y}\right)$. If $P(Y \mid X)$ exists then

$$
E(f \circ Y \mid X)(\xi)=\int_{T} f(\eta) P(Y \mid X)(d \eta, \xi) \quad \text { for a.e. } \xi \in\left(S, \Im, P_{X}\right) .
$$

Proof. Consider the case where $f=\chi_{G}$ for some $G \in(\mathfrak{S}$. Then for every $F \in \mathfrak{F}$ we have by (2.3)

$$
\begin{aligned}
& \int_{F} E(f \circ Y \mid X)(\xi) P_{X}(d \xi)=\int_{X^{-1}(F)} \chi_{G}(Y(\omega)) P(d \omega) \\
& =P\left(Y^{-1}(G) \cap X^{-1}(F)\right) .
\end{aligned}
$$

On the other hand, by $2^{\circ}$ and then $1^{\circ}$ of Definition 3 ,

$$
\begin{aligned}
\int_{T} f(\eta) P(Y \mid X)(d \eta, \xi) & =\int_{T} \chi_{G}(\eta) P(Y \mid X)(d \eta, \xi) \\
=P(Y \mid X)(G, \xi) & =P(Y \in G \mid X)(\xi)
\end{aligned}
$$

so that by (2.2) we have

$$
\int_{F}\left\{\int_{T} f(\eta) P(Y \mid X)(d \eta, \xi)\right\} P_{X}(d \xi)=P\left(Y^{-1}(G) \cap X^{-1}(F)\right) .
$$

Thus the left side of (2.5) is equal to that of (2.6) for every $F \in \mathfrak{F}$ so that (2.4) holds in this case.

Now that (2.4) holds when $f$ is the characteristic function of a member of (S) we can follow the usual procedure in integration theory to show that (2.4) holds for nonnegative simple functions on $T$, nonnegative (S-measurable function on $T$ and finally real valued (s)measurable functions on $T$. Since $f \in L_{1}\left(T, \mathbb{S}, P_{Y}\right)$, both sides of (2.4) always exist and are finite. In passing from nonnegative simple functions on $T$ to nonnegative (S-measurable function on $T$ we use the Monotone Convergence Theorem for the conditional expectation which states that if $\left\{Z_{n}, n=1,2, \cdots\right\} \subset L_{1}(\Omega, \mathfrak{B}, P)$ and $Z_{n}(\omega) \uparrow Z_{0}(\omega)$ for a.e. 


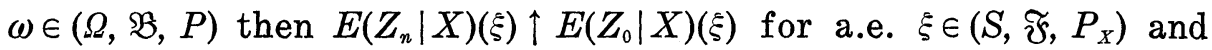
which can be proved readily.

Proposition 2. Let $\sigma(\mathfrak{F} \times(\mathbb{S})$ be the $\sigma$-algebra of subsets of $S \times T$ generated by the semialgebra $\mathfrak{F} \times$ \&s and let $P_{[X, Y]}$ be the probability measure on $(S \times T, \sigma(\Im) \times(S))$ determined by the measurable transformation $[X, Y]$ of $(\Omega, \mathfrak{B})$ into $(S \times T, \sigma(\widetilde{\mho} \times(\mathfrak{S}))$. Let $f$ be a measurable transformation of $\left(S \times T, \sigma(\mathfrak{F} \times(\mathfrak{S}))\right.$ into $\left(R^{1}, \mathfrak{B}^{1}\right)$. If $P(Y \mid X)$ exists then

$$
\begin{array}{r}
E(f \circ[X, Y])=\int_{S \times T} f(\xi, \eta) P_{[X, Y]}(d(\xi, \eta)) \\
\quad=\int_{S}\left\{\int_{T} f(\xi, \eta) P(Y \mid X)(d \eta, \xi)\right\} P_{X}(d \xi)
\end{array}
$$

in the sense that the existence of any member in (2.7) implies that of the other and the equality of all.

Proof. The first equality in (2.7) is standard. Let us prove the second. Consider the case where

$$
f(\xi, \eta)=\chi_{F \times G}(\xi, \eta)=\chi_{F}(\xi) \chi_{G}(\eta) \text { for }(\xi, \eta) \in S \times T
$$

where $F \in \mathfrak{F}$ and $G \in \mathbb{S}$. Then by $2^{\circ}$ and $1^{\circ}$ of Definition 3 and by (2.2)

$$
\begin{aligned}
& \int_{S}\left\{\int_{T} f(\xi, \eta) P(Y \mid X)(d \eta, \xi)\right\} P_{X}(d \xi) \\
& \quad=\int_{S} \chi_{F}(\xi)\left\{\int_{T} \chi_{G}(\eta) P(Y \mid X)(d \eta, \xi)\right\} P_{X}(d \xi) \\
& \quad=\int_{S} \chi_{F}(\xi) P(Y \mid X)(G, \xi) P_{X}(d \xi) \\
& \quad=\int_{F} P(Y \in G \mid X)(\xi) P_{X}(d \xi) \\
& \quad=P\left(Y^{-1}(G) \cap X^{-1}(F)\right)
\end{aligned}
$$

while

$$
\begin{array}{rl}
\int_{S \times T} & f(\xi, \eta) P_{[X, Y]}(d(\xi, \eta)) \\
& =\int_{S \times T} \chi_{F \times G}(\xi, \eta) P_{[X, Y]}(d(\xi, \eta)) \\
& =P_{[X, Y]}(F \times G) \\
& =P\{\omega \in \Omega ; X(\omega) \in F \text { and } Y(\omega) \in G\} \\
& =P\left(Y^{-1}(G) \cap X^{-1}\left(F^{\prime}\right)\right)
\end{array}
$$

so that the second equality in (2.7) holds for this particular case. 
We then proceed as in the proof of the Fubini Theorem to an arbitrary real valued $\sigma(\mathfrak{F} \times(\mathbb{S})$-measurable function $f$ on $S \times T$ to complete the proof.

Proposition 3. Let $Z$ be a real valued random variable on $(\Omega$, $\mathfrak{B}, P)$ with $E(|Z|)<\infty$ and let $g$ be a measurable transformation of $(S, \mathfrak{\mho})$ into $\left(R^{1}, \mathfrak{B}^{1}\right)$. Then

$$
E[(g \circ X) Z]=\int_{S} g(\xi) E(Z \mid X)(\xi) P_{X}(d \xi)
$$

in the sense that the existence of one side implies that of the other and the equality of the two.

Proof. Let us define a set function $\Phi$ on $\mathfrak{B}$ by

$$
\Phi(B)=\int_{B} Z(\omega) P(d \omega) \quad \text { for } \quad B \in \mathfrak{B} .
$$

Since $E(|Z|)<\infty, \Phi$ is a finite signed measure on $(\Omega, \mathfrak{B})$ which is absolutely continuous with respect to $P$ and has $Z$ as its RadonNikodym derivative with respect to $P$. Thus for the real valued random variables $g \circ X$ and $Z$ on $(\Omega, \Re, P)$ we have

$$
E[(g \circ X) Z]=\int_{\Omega} g(X(\omega)) Z(\omega) P(d \omega)=\int_{\Omega} g(X(\omega)) \Phi(d \omega)
$$

in the sense that the existence of one member implies that of the others and the equality of all. Then, to prove (2.8) it suffices to show that

$$
\int_{\Omega} g(X(\omega)) \Phi(d) \omega=\int_{S} g(\xi) E(Z \mid X)(\xi) P_{X}(d \xi)
$$

in the sense that the existence of one side implies that of the other and the equality of the two.

Let us consider the case where $g=\chi_{F}$ for some $F \in \mathfrak{F}$. Then

$$
\begin{gathered}
\int_{\Omega} g(X(\omega)) \Phi(d \omega)=\int_{\Omega} \chi_{F}(X(\omega)) \Phi(d \omega)=\int_{X^{-1}(F)} \Phi(d \omega) \\
=\int_{X^{-1}(F)} Z(\omega) P(d \omega)=\int_{F} E(Z \mid X)(\xi) P_{X}(d \xi) \\
=\int_{S} g(\xi) E(Z \mid X)(\xi) P_{X}(d \xi)
\end{gathered}
$$

by (2.3) so that (2.9) holds. Following the standard procedure in integration theory we proceed from this particular case to nonnegative simple functions on $S$, nonnegative $\widetilde{\mho}$-measurable functions on $S$ and finally real valued $\mathfrak{\mho}$-measurable functions on $S$ to complete 
the proof.

3. Inversion of conditional expectations. It is well-known that if the characteristic function $\varphi$ of a distribution function $F$ on $R^{1}$ is $m_{L}$-integrable on $R^{1}$, then $F$ is absolutely continuous and

$$
F^{\prime}(\xi)=\frac{1}{2 \pi} \int_{R^{1}} e^{-i(\xi, \eta)} \varphi(\eta) m_{L}(d \eta) \quad \text { for } \quad \text { a.e. } \xi \in\left(R^{1}, \mathfrak{B}^{1}, m_{L}\right) \text {. }
$$

Let $\Phi$ be a finite measure on $\left(R^{k}, \mathfrak{B}^{k}\right)$ and let $\varphi$ be its characteristic function, i.e.,

$$
\varphi(\eta)=\int_{R^{k}} e^{i(\xi, \eta)} \Phi(d \xi) \quad \text { for } \quad \eta \in R^{k}
$$

where $(\xi, \eta)=\sum_{j=1}^{k} \xi_{j} \eta_{j}$. According to the Lévy-Haviland Inversion Theorem (see [3] and [4])

$$
\begin{aligned}
& \int_{R^{k}} \prod_{j=1}^{k} \tilde{\chi}_{a_{j}, b_{j}}\left(\xi_{j}\right) \Phi(d \xi) \\
& \quad=\lim _{h \rightarrow \infty} \frac{1}{(2 \pi)^{k}} \int_{C_{h}} \prod_{j=1}^{k} \frac{e^{-i b_{j} \eta_{j}}-e^{-i a_{j} \eta_{j}}}{-i \eta_{j}} \varphi(\eta) m_{L}(d \eta)
\end{aligned}
$$

for any $a_{j}, b_{j} \in R^{1}, a_{j}<b_{j}, j=1,2, \cdots, k$, where

$$
C_{h}=(-h, h) \times \cdots \times(-h, h) \subset R^{k} \quad \text { with } h>0,
$$

and the modified characteristic function $\tilde{\chi}_{a j, b j}$ is defined by

$$
\tilde{\chi}_{a_{j}, b_{j}}\left(\eta_{j}\right)=\left\{\begin{array}{lll}
1 & \text { for } & \eta_{j} \in\left(a_{j}, b_{j}\right) \\
0 & \text { for } & \eta_{j} \in\left[a_{j}, b_{j}\right]^{c} \\
\frac{1}{2} & \text { for } & \eta_{j}=a_{j} \text { and for } \eta_{j}=b_{j} .
\end{array}\right.
$$

From (3.2) we derive the following:

THEOREM 1. If the characteristic function $\varphi$ of a finite measure $\Phi$ on $\left(R^{k}, \mathfrak{B}^{k}\right)$ is $m_{L^{-}}$integrable on $R^{k}$, then $\Phi$ is absolutely continuous with respect to $m_{L}$ on $\left(R^{k}, \mathfrak{B}^{k}\right)$ and a version of the Radon-Nikodym derivative of $\Phi$ with respect to $m_{L}$ is given by

$$
\frac{d \Phi}{d m_{L}}(\xi)=\frac{1}{(2 \pi)^{k}} \int_{R^{k}} e^{-i(\xi, \eta)} \varphi(\eta) m_{L}(d \eta) \quad \text { for } \quad \xi \in R^{k} .
$$

Proof. Since the $j$ th factor of the product in the integrand on the right side of (3.2) is a bounded continuous function of $\eta_{j} \in$ $R^{1}$, if we assume the $m_{L}$-integrability of $\varphi$ on $R^{k}$ then the integrand on the right side of (3.2) is $m_{L}$-integrable on $R^{k}$ so that (3.2) reduces to 


$$
\begin{aligned}
& \int_{R^{k}} \prod_{j=1}^{k} \widetilde{X}_{a_{j}, b_{j}}\left(\xi_{j}\right) \Phi(d \xi) \\
& \quad=\frac{1}{(2 \pi)^{k}} \int_{R^{k}} \prod_{j=1}^{k} \frac{e^{-i b_{j} \eta_{j}}-e^{-i a_{j} \eta_{j}}}{-i \eta_{j}} \varphi(\eta) m_{L}(d \eta) .
\end{aligned}
$$

To show that $\Phi$ is absolutely continuous with respect to $m_{L}$ on $\left(R^{k}, \mathfrak{B}^{k}\right)$ let $A \in \mathfrak{B}^{k}$ and $m_{L}(A)=0$. We proceed to show that $\Phi(A)=$ 0 . Let $\mathfrak{A}$ be the algebra of subsets of $R^{k}$ which are unions of finitely many disjoint half open and half closed intervals $\left(a_{1}, b_{1}\right] \times \cdots$ $\times\left(a_{k}, b_{k}\right]$ in $R^{k}$. Then the $\sigma$-algebra of subsets of $R^{k}$ generated by $\mathfrak{A}$ is precisely our $\mathfrak{B}^{k}$. Let $\varepsilon>0$ be arbitrarily given. Since $\Phi$ is a finite measure on $\mathfrak{B}^{k}$ and since $m_{L}(A)$ is finite (in fact equal to zero), $\left(\Phi+m_{L}\right)(A)$ is finite so that there exists some $B \in \mathfrak{X}$ such that

$$
\left(\Phi+m_{L}\right)(A \Delta B)<\varepsilon
$$

where $A \Delta B$ is the symmetric difference between $A$ and $B$. Now (3.7) implies that $\Phi(A \Delta B)<\varepsilon$ so that

$$
\Phi(A) \leqq \Phi(B)+\Phi(A \Delta B)<\Phi(B)+\varepsilon .
$$

It also implies that $m_{L}(A \Delta B)<\varepsilon$ so that in view of $m_{L}(A)=0$ we have

$$
m_{L}(B)<\varepsilon .
$$

Since $B$ is the union of finitely many, say $m$, disjoint half open half closed intervals, there exist $m$ open intervals $B^{(n)}, n=1,2, \cdots, m$, such that

$$
B \subset \bigcup_{n=1}^{m} B^{(n)} \quad \text { and } \quad m_{L}(B)<\sum_{n=1}^{m} m_{L}\left(B^{(n)}\right)<\varepsilon .
$$

Let each $B^{(n)}$ be given as

$$
B^{(n)}=\zeta^{(n)}+C^{(n)}
$$

where

$$
\zeta^{(n)} \in R^{k} \text { and } C^{(n)}=\left(-h_{1}^{(n)}, h_{1}^{(n)}\right) \times \cdots \times\left(-h_{k}^{(n)}, h_{k}^{(n)}\right) \subset R^{k} .
$$

In view of the openness of $C^{(n)}$ and the definition of $\tilde{\chi}_{a_{j}, b_{j}}$ by (3.4) we have from (3.6)

$$
\begin{aligned}
\Phi\left(\zeta^{(n)}\right. & \left.+C^{(n)}\right) \\
& \leqq \frac{1}{(2 \pi)^{k}} \int_{R^{k}} \prod_{j=1}^{k} \frac{e^{-i\left(\zeta_{j}^{(n)}+h_{j}^{(n)} \eta_{j}\right.}-e^{-i\left(\zeta_{j}^{(n)}-h_{j}^{(n)} \eta_{j}\right)}}{-i \eta_{j}} \\
= & \frac{m_{L}\left(C^{(n)}\right)}{(2 \pi)^{k}} \int_{R^{k}} \prod_{j=1}^{k} \frac{\sin \eta_{j} h_{j}^{(n)}}{\eta_{j} h_{j}^{(n)}} e^{-i \zeta_{j}^{(n)} \eta_{j}} \varphi(\eta) m_{L}(d \eta) .
\end{aligned}
$$


Since $\left|\left(\eta_{j} h_{j}^{(n)}\right)^{-1} \sin \eta_{j} h_{j}^{(n)}\right| \leqq 1$ for $\eta_{j} \in R^{1}$ and since $m_{L}\left(C^{(n)}\right)=m_{L}\left(B^{(n)}\right)$ we have

$$
\Phi\left(B^{(n)}\right)=\Phi\left(\zeta^{(n)}+C^{(n)}\right) \leqq \frac{m_{L}\left(B^{(n)}\right)}{(2 \pi)^{k}} \int_{R^{k}}|\varphi(\eta)| m_{L}(d \eta)
$$

From (3.9) and (3.13) we obtain

$$
\Phi(B) \leqq \sum_{n=1}^{m} \Phi\left(B^{(n)}\right) \leqq \frac{\varepsilon}{(2 \pi)^{k}} \int_{R^{k}}|\varphi(\eta)| m_{L}(d \eta)
$$

Using (3.14) in (3.8) we have

$$
\Phi(A)<\varepsilon\left\{\frac{1}{(2 \pi)^{k}} \int_{R^{k}}|\varphi(\eta)| m_{L}(d \eta)+1\right\} .
$$

From the arbitrariness of $\varepsilon>0$ we have $\Phi(A)=0$. This proves the absolute continuity of $\Phi$ with respect to $m_{L}$ on $\left(R^{k}, \mathfrak{B}^{k}\right)$.

To obtain the Radon-Nikodym derivative of $\Phi$ with respect to $m_{L}$ on $\left(R^{k}, \mathfrak{B}^{k}\right)$, let us observe first that the absolute continuity of $\Phi$ with respect to $m_{L}$ implies that the $\Phi$ measure of the boundary of the open interval $C^{(n)}$ in (3.11) is equal to zero. Thus in (3.12) the strict equality actually holds. If we apply this improved (3.12) to $\zeta+C_{h}$ where $\zeta$ is an arbitrary point in $R^{k}$ and $C_{h}$ is an open interval in $R^{k}$ as given by (3.3) then we have

$$
\begin{aligned}
& \int_{\zeta+C_{h}} \frac{d \Phi}{d m_{L}}(\xi) m_{L}(d \xi)=\Phi\left(\zeta+C_{h}\right) \\
& \quad=\frac{m_{L}\left(C_{h}\right)}{(2 \pi)^{k}} \int_{R^{k}} \prod_{j=1}^{k} \frac{\sin \eta_{j} h}{\eta_{j} h} e^{-i \zeta_{j} \eta_{j}} \varphi(\eta) m_{L}(d \eta) .
\end{aligned}
$$

Let $h \rightarrow 0$ on both sides of (3.15). On the one hand we have

$$
\begin{aligned}
\lim _{h \rightarrow 0} & \frac{1}{m_{L}\left(C_{h}\right)} \int_{\zeta+C_{h}} \frac{d \Phi}{d m_{L}}(\xi) m_{L}(d \xi) \\
& =\frac{d \Phi}{d m_{L}}(\zeta), \text { for a.e. } \zeta \in\left(R^{k}, \mathfrak{B}^{k}, m_{L}\right)
\end{aligned}
$$

and on the other hand by the Dominated Convergence Theorem

$$
\begin{gathered}
\lim _{h \rightarrow 0} \frac{1}{(2 \pi)^{k}} \int_{R^{k}} \prod_{j=1}^{k} \frac{\sin \eta_{j} h}{\eta_{j} h} e^{-i \zeta_{j} \eta} \varphi(\eta) m_{L}(d \eta) \\
=\frac{1}{(2 \pi)^{k}} \int_{R^{k}} e^{-(\zeta, \eta)} \varphi(\eta) m_{L}(d \eta) .
\end{gathered}
$$

Using (3.16) and (3.17) in (3.15) we have

$$
\frac{d \Phi}{d m_{L}}(\zeta)=\frac{1}{(2 \pi)^{k}} \int_{R^{k}} e^{-(\zeta, \eta)} \varphi(\eta) m_{L}(d \eta) \text { for a.e. } \zeta \in\left(R^{k}, \mathfrak{B}^{k}, m_{L}\right) \text {. }
$$


This completes the proof of the theorem.

By means of Proposition 2 and Theorem 1 our inversion theorem for conditional expectation can be derived now.

THEOREM 2. Let $Y$ be a real valued random variable on a probability space $(\Omega, \mathfrak{B}, P)$ with $E(|Y|)<\infty$ and let $X$ be a $k$-dimensional random vector i.e., a measurable transformation of $(\Omega, \mathfrak{B})$ into $\left(R^{k}\right.$, $\left.\mathfrak{B}^{k}\right)$. Assume that the probability distribution $P_{X}$ of $X$ is absolutely continuous with respect to $m_{L}$ on $\left(R^{k}, \mathfrak{B}^{k}\right)$. If $E\left[e^{i(u, X)} Y\right]$ is a $m_{L^{-}}$ integrable function of $u$ on $R^{k}$ then $a$ version of the conditional expectation of $Y$ given $X, E(Y \mid X)$, is given by

$$
E(Y \mid X)(\xi) \frac{d P_{X}}{d m_{L}}(\xi)=\frac{1}{(2 \pi)^{k}} \int_{R^{k}} e^{-i(u, \xi)} E\left[e^{i(u, X)} Y\right] m_{L}(d u)
$$

for $\xi \in R^{k}$.

Proof. Since $Y$ is a measurable transformation of $(\Omega, \mathfrak{B})$ into $\left(R^{1}, \mathfrak{B}^{1}\right)$ which is a Borel space, the regular conditional distribution of $Y$ given $X, P(Y \mid X)$, exists. With fixed $u \in R^{k}$ consider a complex valued function $f$ on $R^{k} \times R^{1}$ defined by

$$
f(\xi, \eta)=e^{i(u, \xi)} \eta \quad \text { for } \xi \in R^{k} \text { and } \eta \in R^{1} .
$$

Applying (2.7) of Proposition 2 and (2.4) of Proposition 1 to the real and the imaginary parts of $f$ we obtain

$$
\begin{aligned}
E\left[e^{i(u, X)} Y\right]= & \int_{R^{k}} e^{i(u, \xi)}\left\{\int_{R^{1}} \eta P(Y \mid X)(d \eta, \xi)\right\} P_{X}(d \xi) \\
& \int_{R^{k}} e^{i(u, \xi)} E(Y \mid X)(\xi) P_{X}(d \xi) .
\end{aligned}
$$

Consider a set function $\Phi$ defined on $\mathfrak{B}^{k}$ by

$$
\Phi(F)=\int_{F} E(Y \mid X)(\xi) P_{X}(d \xi) \quad \text { for } \quad F \in \mathfrak{B}^{k}
$$

Since $E(Y \mid X)$ is $P_{X}$ integrable on $R^{k}, \Phi$ is a finite signed measure on $\left(R^{k}, \mathfrak{B}^{k}\right)$ which is absolutely continuous with respect to $P_{X}$ on $\left(R^{k}, \mathfrak{B}^{k}\right)$ and has $E(Y \mid X)$ as its Radon-Nikodym derivative with respect to $P_{X}$. According to (3.19), $E\left[e^{i(u, X)} Y\right], u \in R^{k}$, is the characteristic function of $\Phi$. Under the hypothesis of the theorem, this characteristic function is $m_{L}$-integrable over $R^{k}$. Applying Theorem 1 to the positive and the negative part of $\Phi$ we obtain 


$$
\begin{aligned}
& \frac{d \Phi}{d m_{L}}(\xi) \\
& \quad=\frac{1}{(2 \pi)^{k}} \int_{R^{k}} e^{-i(u, \xi)} E\left[e^{i(u, X)} Y\right] m_{L}(d u) \text { for a.e. } \xi \in\left(R^{k}, \mathfrak{B}^{k}, m_{L}\right)
\end{aligned}
$$

Since by (3.20)

$$
\frac{d \Phi}{d m_{L}}(\xi)=E(Y \mid X)(\xi) \frac{d P_{X}}{d m_{L}}(\xi) \quad \text { for a.e. } \xi \in\left(R^{k}, \mathfrak{B}^{k}, m_{L}\right)
$$

we have (3.18).

\section{REFERENCES}

1. L. Breiman, Probability, Addison-Wesley, Reading, Mass., 1968.

2. J. L. Doob, Stochastic Processes, Wiley, New York, 1953.

3. E. K. Haviland, On the inversion formula for Fourier-Stieltjes transforms in more than one dimension, Amer. J. Math., 57 (1935), 94-100.

4. K. Itô, Probability Theory (in Japanese), Iwanani, Tokyo, 1953.

Received August 13, 1973.

UNiversity of CALIFornia, Irvine 


\section{PACIFIC JOURNAL OF MATHEMATICS}

EDITORS

RICHARD ARens (Managing Editor)

University of California

Los Angeles, California 90024

\section{R. A. Beaumont}

University of Washington

Seattle. Washington 98105

\section{J. DugundjI}

Department of Mathematics University of Southern California Los Angeles, California 90007

D. Gilbarg and J. Milgram

Stanford University

Stanford. California 94305

\section{ASSOCIATE EDITORS}
E. F. BECKENBACH
B. H. NEUMANN
F. WOLF
K. Yoshida

\section{SUPPORTING INSTITUTIONS}

\author{
UNIVERSITY OF BRITISH COLUMBIA \\ CALIFORNIA INSTITUTE OF TECHNOLOGY \\ UNIVERSITY OF CALIFORNIA \\ MONTANA STATE UNIVERSITY \\ UNIVERSITY OF NEVADA \\ NEW MEXICO STATE UNIVERSITY \\ OREGON STATE UNIVERSITY \\ UNIVERSITY OF OREGON \\ OSAKA UNIVERSITY
}

\author{
UNIVERSITY OF SOUTHERN CALIFORNIA \\ STANFORD UNIVERSITY \\ UNIVERSITY OF TOKYO \\ UNIVERSITY OF UTAH \\ WASHINGTON STATE UNIVERSITY \\ UNIVERSITY OF WASHINGTON

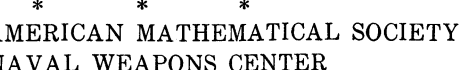

The Supporting Institutions listed above contribute to the cost of publication of this Journal, but they are not owners or publishers and have no responsibility for its content or policies.

Mathematical papers intended for publication in the Pacific Journal of Mathematics should be in typed form or offset-reproduced, (not dittoed), double spaced with large margins. Underline Greek letters in red, German in green, and script in blue. The first paragraph or two must be capable of being used separately as a synopsis of the entire paper. Items of the bibliography should not be cited there unless absolutely necessary, in which case they must be identified by author and Journal, rather than by item number. Manuscripts, in duplicate if possible, may be sent to any one of the four editors. Please classify according to the scheme of Math. Rev. Index to Vol. 39. All other communications to the editors should be addressed to the managing editor, or Elaine Barth, University of California, Los Angeles, California, 90024.

100 reprints are provided free for each article, only if page charges have been substantially paid Additional copies may be obtained at cost in multiples of 50 .

The Pacific of Journal Mathematics is issued monthly as of January 1966. Regular subscription rate: $\$ 72.00$ a year (6 Vols., 12 issues). Special rate: $\$ 36.00$ a year to individual members of supporting institutions.

Subscriptions, orders for back numbers, and changes of address should be sent to Pacific Journal of Mathematics, 103 Highland Boulevard, Berkeley, California, 94708.

\section{PUBLISHED BY PACIFIC JOURNAL OF MATHEMATICS, A NON-PROFIT CORPORATION}

Printed at Kokusai Bunken Insatsusha (International Academic Printing Co., Ltd.), 270, 3-chome Totsuka-cho. Shinjuku-ku, Tokyo 160. Japan.

Copyright (C) 1973 by Pacific Journal of Mathematics Manufactured and first issued in Japan 


\section{Pacific Journal of Mathematics}

\section{Vol. 52, No. $2 \quad$ February, 1974}

Harm Bart, Spectral properties of locally holomorphic vector-valued functions .....

J. Adrian (John) Bondy and Robert Louis Hemminger, Reconstructing infinite

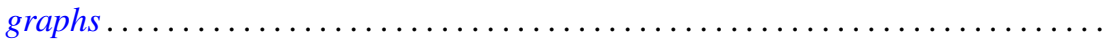

Bryan Edmund Cain and Richard J. Tondra, Biholomorphic approximation of planar

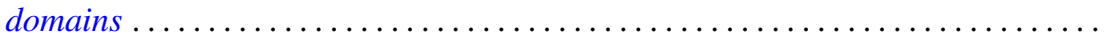

Richard Carey and Joel David Pincus, Eigenvalues of seminormal operators,

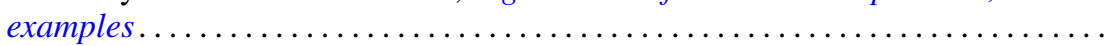

Tyrone Duncan, Absolute continuity for abstract Wiener spaces . . . . . . . . . . . . Joe Wayne Fisher and Louis Halle Rowen, An embedding of semiprime

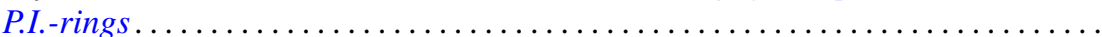

Andrew S. Geue, Precompact and collectively semi-precompact sets of semi-precompact continuous linear operators. . . . . . . . . . . . . . . .

Charles Lemuel Hagopian, Locally homeomorphic $\lambda$ connected plane continua ..... . Darald Joe Hartfiel, A study of convex sets of stochastic matrices induced by

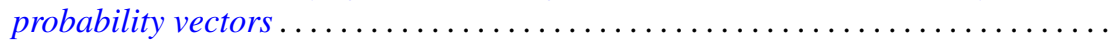

Yasunori Ishibashi, Some remarks on high order derivations $\ldots \ldots \ldots \ldots \ldots \ldots \ldots$ Donald Gordon James, Orthogonal groups of dyadic unimodular quadratic forms.

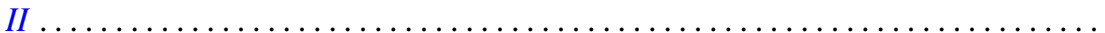

Geoffrey Thomas Jones, Projective pseudo-complemented semilattices . . . . . . . . . Darrell Conley Kent, Kelly Denis McKennon, G. Richardson and M. Schroder,

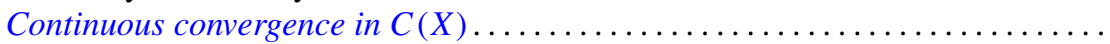

J. J. Koliha, Some convergence theorems in Banach algebras ...

Tsang Hai Kuo, Projections in the spaces of bounded linear oper

George Berry Leeman, Jr., A local estimate for typically real functions . .

475

Andrew Guy Markoe, A characterization of normal analytic spaces by the

homological codimension of the structure sheaf .........

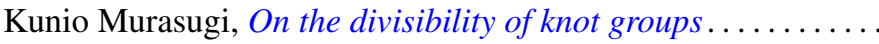

John Phillips, Perturbations of type I von Neumann algebras.

Billy E. Rhoades, Commutants of some quasi-Hausdorff matrices . .

David W. Roeder, Category theory applied to Pontryagin duality

Maxwell Alexander Rosenlicht, The nonminimality of the differential closure .

Peter Michael Rosenthal, On an inversion theorem for the general Mehler-Fock transform pair.

Alan Saleski, Stopping times for Bernoulli automorphisms

John Herman Scheuneman, Fundamental groups of compact complete locally affine complex surfaces. II. ........................

Vashishtha Narayan Singh, Reproducing kernels and operators with a cyclic vector. I. .

Peggy Strait, On the maximum and minimum of partial sums of random variables.

J. L. Brenner, Maximal ideals in the near ring of polynomials modulo 2 .

Ernst Gabor Straus, Remark on the preceding paper: "Ideals in near rings of polynomials over a field" ..........................

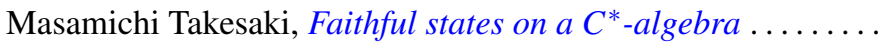

R. Michael Tanner, Some content maximizing properties of the regular simplex.

Andrew Bao-hwa Wang, An analogue of the Paley-Wiener theorem for certain

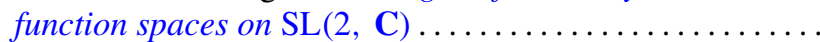

